\title{
Self-Gravitational Instability of an Isothermal Gaseous Slab Under High External Pressure
}

\author{
Michihisa Umekawa \\ Astrophysics Laboratory, Department of Physics, Faculty of Science, \\ Chiba University, c/o Prof. Ryoji Matsumoto, 1-33 Yayoi-cho, \\ Inage-ku, Chiba 263-8522, Japan \\ Ryoji Matsumoto \\ Department of Physics, Faculty of Science, Chiba University, 1-33 \\ Yayoi-cho, Inage-ku, Chiba 263-8522, Japan \\ Shigeki Miyaji \\ Department of Physics, Faculty of Science, Chiba University, 1-33 \\ Yayoi-cho, Inage-ku, Chiba 263-8522, Japan \\ Tatsuo Yoshida \\ Department of Physics, Faculty of Science, Ibaraki University, 2-1-1 \\ Bunkyo, Mito, Ibaraki 310-8512, Japan
}

\section{Introduction}

In active massive star forming regions such as Orion and the Galactic center, the self-gravitational instability of a magnetized gaseous slab plays an important role as a trigger of star formation. In such high external pressure regions, the incompressible mode of self-gravitational instability (Elmegreen \& Elmegreen 1978; Lubow \& Pringle 1992) becomes dominant. Based on two-dimensional hydrodynamical simulations, Umekawa et al. (1999) proposed "Star formation by merging of the Jeans stable clumps" in a pressure bounded slab. In a magnetized slab confined by external pressure, Nagai et al. (1998) showed by linear analysis that the slab fragments to filaments parallel to the magnetic field lines. Here, we show by nonlinear three-dimensional MHD simulations that the filaments further fragment to Jeans stable clumps.

\section{Models and Numerical Results}

We use a three-dimensional MHD code in Cartesian coordinates based on a modified Lax-Wendroff scheme. We used the ICCG (Meijerink \& Vorst 1981) method to implement the Poisson equation for self-gravity. Here we report the numerical model shown in table 1 .

The result of numerical simulations is shown in figure 1. The left panel shows the magnetic field lines and isosurface of density. We confirmed the expectation from the linear theory (Nagai et al. 1998) that when external pressure is large, the magnetized gaseous slab fragments to filaments parallel to the mag- 
Table 1. Model parameters for numerical simulations

\begin{tabular}{lcccc}
\hline \hline Model name & $\beta$ & $P_{\text {ext }}$ & Grids & W \\
\hline 3 & 1.0 & 0.94 & $65 \times 33 \times 75$ & 5.0 \\
\hline \hline
\end{tabular}

NOTE: $\beta, P_{\text {ext }}$, and $\mathrm{W}$ are the ratio of thermal to magnetic pressure at mid-plane, external pressure of the gaseous slab normalized by mid-plane pressure, and length of simulation region normalized by the linearly most unstable wavelength.
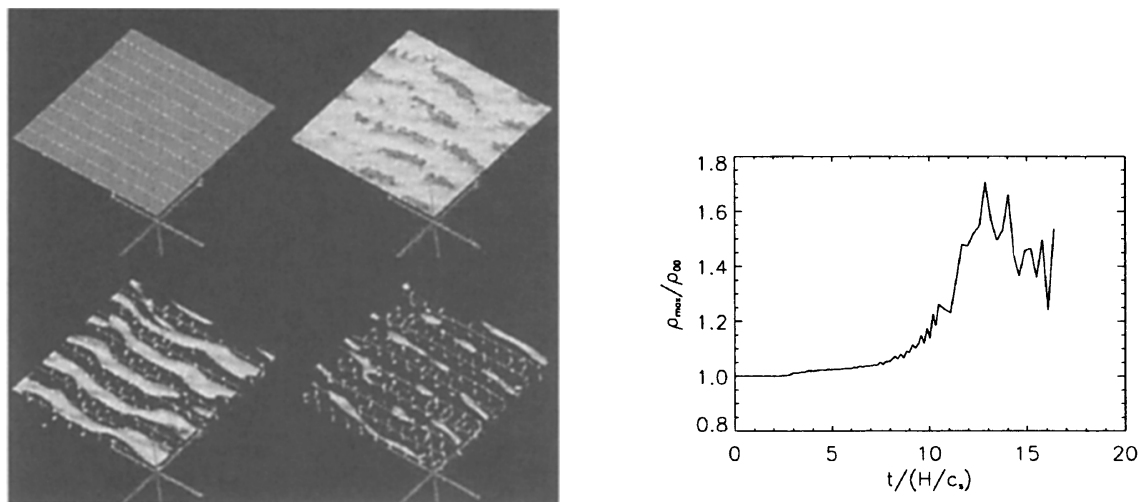

Figure 1. Results of numerical simulation of model 3. (Left) The time evolution, $t=0.0 \mathrm{H} / c_{\mathrm{s}}, 9.0 \mathrm{H} / c_{\mathrm{s}}, 12.0 \mathrm{H} / c_{\mathrm{s}}$, and $15.0 \mathrm{H} / c_{\mathrm{s}}$, respectively, where $H$ is scale height and $c_{\mathrm{s}}$ is sound speed. Gray surfaces show isosurface of density $\rho=0.94 \rho_{00}$ and white surface is $\rho=1.0 \rho_{00}$, where $\rho_{00}$ is initial mid-plane density of the gaseous slab. White curves show magnetic field lines. Vectors show velocity distribution. (Right) The time evolution of maximum density normalized by $\rho_{00}$.

netic field lines. Subsequently, the filaments fragment to small clumps. These clumps are not in the collapsing stage, because their mass is smaller than the Jeans mass. The results of our 3-D non-linear MHD simulation support earler work based on 2-D linear analysis that star formation proceeds by the merging of Jeans-stable clumps.

\section{References}

Elmegreen, B. G., \& Elmegreen, D. M. 1978, ApJ, 220, 1051

Lubow, S. H., \& Pringle, J. E. 1993, MNRAS, 263, 701

Meijerink, J. A., \& van der Vorst, H. A. 1981, J. Comput. Phys., 44, 134

Nagai T., Inutsuka S., \& Miyama S. M. 1998, ApJ, 506, 306

Umekawa, M., Matsumoto, R., Miyaji, S., \& Yoshida, T. 1999, PASJ, 51, 625 